\title{
Preliminary Outcomes 1 Year after Laparoscopic Sleeve Gastrectomy Based on Bariatric Analysis and Reporting Outcome System (BAROS)
}

\author{
Maciej Bobowicz • Andrzej Lehmann • \\ Michal Orlowski • Pawel Lech • Maciej Michalik
}

Published online: 15 April 2011

(C) The Author(s) 2011. This article is published with open access at Springerlink.com

\begin{abstract}
Background The aim of this study was to assess outcomes of laparoscopic sleeve gastrectomy (LSG) as a stand-alone bariatric operation according to the Bariatric Analysis and Reporting Outcome System (BAROS).

Methods Out of 112 patients included and operated on initially, 84 patients (F/M, 63:21) were followed up for 1456 months (mean 22 \pm 6.75 ). Patients lost to follow-up did not attend scheduled follow-up visits or they have withdrawn their consent. Mean age was 39 years (range 17-67; $\mathrm{SD} \pm 12.09$ ) with mean initial BMI $44.62 \mathrm{~kg} / \mathrm{m}^{2}$ (range 29.39-82.8; $\mathrm{SD} \pm 8.17)$. Statistical significance was established at the $p<0.05$ level.

Results Mean operative time was $61 \mathrm{~min}(30-140 \mathrm{~min})$ with mean hospital stay of 1.37 days $(0-4 ; \mathrm{SD} \pm 0.77)$. Excellent global BAROS outcome was achieved in 13\% of patients, very good in $30 \%$, good in $34.5 \%$, fair $9.5 \%$ and failure in $13 \%$ patients 12 months after surgery. Females achieved significantly better outcomes than males with the mean $46.5 \%$ of excess weight loss (EWL) versus $35.3 \%$ of EWL at 12 months $(p=0.02)$. The mean percentage of excess weight loss (\%EWL) was $43.6 \%$ at 12 months and $46.6 \%$ at 24 months. Major surgical complication rate was $7.1 \%$; minor surgical complication rate $8.3 \%$. There was one conversion $(1.2 \%)$ due to the massive bleeding. Comorbidities improved or resolved in numerous patients:
\end{abstract}

M. Bobowicz · A. Lehmann • M. Orlowski · P. Lech • M. Michalik General and Vascular Surgery Department, Ceynowa Hospital, Jagalskiego 10, Wejherowo 84-200, Poland

M. Bobowicz $(\bowtie)$

Department of Surgical Oncology, Medical University of Gdansk, Debinki 7, Gdansk 80-211, Poland

e-mail: mbobowicz@gumed.edu.pl arterial hypertension in $62 \%$, diabetes mellitus in $68.3 \%$, respectively.

Conclusions Presented LSG series shows that the LSG as a stand-alone procedure provides acceptable $\% \mathrm{EWL}$ and good global BAROS outcomes. It significantly improves comorbidities as well.

Keywords Laparoscopic sleeve gastrectomy - LSG · Treatment outcomes - Bariatric analysis and reporting outcome system $\cdot$ BAROS $\cdot$ Excess weight loss

\section{Introduction}

Sleeve gastrectomy is one of the restrictive operations used to treat morbid obesity [1]. This operation was first described by Hess in 1988 as a part of the biliopancreatic diversion with duodenal switch (BPD-DS) [2]. In 1999, Gagner performed first laparoscopic sleeve gastrectomy (LSG) also as a part of the BPD-DS [3]. Later, he used LSG as a staged procedure for super morbid obesity to finally use it as a stand-alone procedure. Since its first implementation in 2004 as a stand-alone bariatric operation, LSG was proved to be sufficient and became one of the procedures on the incline.

To assess the results of bariatric treatment, authors use multiple outcome factors such as percentage of excess weight loss, quality of life and complications including postoperative deaths. Some use standardized tools such as SF-36 scale [4], Sickness Impact Profile [5], Quality of Well-Being Scale [6], and finally Bariatric Analysis and Reporting Outcome System (BAROS) [7]. BAROS, developed by Oria and Moorehead, is still the most comprehensive questionnaire; it is also easy to use in daily practice. 
BAROS assesses percentage of excess weight loss (\%EWL), improvement and/or resolution of comorbid conditions, five aspects of quality of life (self-esteem, physical activity, social activity, work, and sexual activity), complications, and reoperations. The final outcome is based on improvement, worsening, or no change in all five listed domains giving the most comprehensive assessment of the treatment results influencing not only the weight changes but also its impact on patients' general health and well being.

The aim of this study was to assess outcomes of laparoscopic sleeve gastrectomy used as a stand-alone procedure for morbid obesity in a single institution in Poland according to BAROS criteria.

\section{Method}

The study was designed as a single-institution, observational study. There was a retrospective data analysis based on the patients' hospital records (baseline data including height, weight, comorbidities, data on operation, and complications) and prompted self-reported data collected over the telephone (changes in time in weight, complications, reoperations, changes in QoL, changes in comorbidities, eating behaviors, and physical activity changes). The patients were included since the introduction of the procedure in the authors' institution. Between 13th March 2008 and 16th December 2009, 112 patients signed informed consent and were initially qualified to participate in the study and underwent LSG operation. All operations were performed by one team of three surgeons following one standard technique. Only the size of bougie was under the surgeons' discretion and was slightly modified depending on the surgeon's preference. Twenty-five percent of patients were lost to follow-up. These patients did not attend scheduled follow-up visits, nor was there current contact data available or they have withdrawn their consent to participate in the study on the later stage when the data was collected in 6-month intervals. The mortality data was checked against the national registry. The remaining 84 patients $(\mathrm{F} / \mathrm{M}, 63: 21)$ were followed-up for 14-56 months (mean 22 \pm 6.75 ). Mean age was 39 years (range 17-67; $\mathrm{SD} \pm 12.09$; 38.7 years for females and 40.1 years for males $(p=0.6)$, with mean initial BMI $44.62 \mathrm{~kg} / \mathrm{m}^{2}$ (range 29.39-82.8; $\mathrm{SD} \pm 8.17)$. Mean BMI significantly differed between genders and was $43 \mathrm{~kg} / \mathrm{m}^{2}$ in females ( $\left.\mathrm{SD} \pm 7.08\right)$ and $49.5 \mathrm{~kg} / \mathrm{m}^{2}$ in males $(\mathrm{SD} \pm 7.35 ; p=0.001)$. Statistical analysis included only patients remaining in the follow-up group to enable outcomes comparison. BAROS questionnaire was used for data collection along with the department's bariatric qualification chart 12 months after surgery to assess preliminary outcomes of laparoscopic sleeve gastrectomy. BAROS incorporates five strategic outcomes of bariatric surgery: \%EWL), resolution or improvement in comorbid conditions, quality of life (the Moorehead-Ardelt Quality of Life Questionnaire, QoL), surgical and medical complications, as well as reoperations all scored as listed below [7].Percentage EWL is stratified in five categories. The weight gain is scored -1 point, $0-24 \%$ EWL gives 0 points, $25-49 \%$ EWL +1 point, $50-74 \%$ EWL +2 points, $>75 \%+3$ points. Comorbidities resolution gives +2 points, its improvement gives +1 point, no change is scored 0 and worsening gives -1 point. Quality of life assessment based on Moorehead-Ardelt QoL Questionnaire evaluates five aspects of live: self-esteem, physical activity, social involvement, ability to work, and interest in sex. Each aspect is evaluated as: much worse, worse, no change, improved, significantly improved receiving $-0.5,-0.25,0,+0.25$, or +0.5 points, respectively. Each major complication deducts one point and a minor complication deducts 0.2 points. Finally, any reoperation deducts one point from the score. Complications were stratified as minor and major according to Oria et al. [7].

Statistical analysis was performed using computer software 'Statistica' 8.0, StatSoft, Krakow, Poland. Statistical significance was established at the $p<0.05$ level for Student's $t$ test and chi-square test.

\section{Results}

\section{Background}

Mean operative time was $61 \mathrm{~min}$ (range $30-140 \mathrm{~min}$ ) with mean hospital stay of 1.37 days (range $0-4 ; \mathrm{SD} \pm$ $0.8)$. Nine out of 84 patients $(10.7 \%)$ were subjected to previous bariatric surgery. Two patients underwent vertical gastric banding, six patients had laparoscopic adjustable gastric banding procedure, and one patient had laparoscopic sleeve gastrectomy. The linear regression model did not show any statistically significant positive or negative impact of having previous bariatric surgery on outcomes of LSG in presented series. Super morbid obesity with $\mathrm{BMI}>50 \mathrm{~kg} / \mathrm{m}^{2}$ was present in $21.4 \%$ $(N=18)$ of patients before the operation and was reduced to $3.6 \%(N=3)$ after the operation. Of the patients, $66.7 \%$ declared having been obese since childhood and $65.5 \%$ having at least one first-degree relative suffering from obesity. Of the patients, $81 \%$ declared having changed their diet with significant calories and carbohydrates intake reduction following the surgery. Thirty-three percent declared increase of physical activity (more than three times a week) and $56 \%$ of patients did not commence any physical activity postoperatively according to selfreported data. 
Table 1 Body mass index before and after surgery

\begin{tabular}{llllrl}
\hline \multicolumn{7}{l}{ BMI $\left(\mathrm{kg} / \mathrm{m}^{2}\right)$} & & \\
\cline { 2 - 5 } & Mean & Minimum & Maximum & SD & $N$ \\
\hline Preoperative & 44.6 & 29.4 & 82.8 & 8.17 & 84 \\
6 Months after surgery & 36.8 & 23.1 & 75.3 & 7.69 & 80 \\
12 Months after surgery & 35.2 & 22.7 & 82.8 & 8.17 & 82 \\
18 Months after surgery & 36.0 & 23.7 & 90.3 & 10.64 & 55 \\
24 Months after surgery & 35.3 & 23.7 & 53.1 & 7.62 & 26 \\
30 Months after surgery & 32.2 & 25.6 & 40.3 & 5.04 & 10 \\
\hline
\end{tabular}

\section{BAROS Data}

Initial mean BMI of $44.6 \mathrm{~kg} / \mathrm{m}^{2}$ has reduced every 6 months to $36.8,35.2,35.9,35.3$, and $32.2 \mathrm{~kg} / \mathrm{m}^{2}$ consecutively at 6 , $12,18,24$, and 30 months after surgery (Table 1).

This corresponded with the mean $36.8 \%$ EWL in the first 6 months and 43.6\% EWL at 12 months, $45.4 \%$ EWL at 18 months, $46.6 \%$ EWL at 24 months, and $51.1 \%$ of EWL at 30 months following surgery. Figure 1 shows changes of the percentage of excess weight loss in time.

Of the patients, $23.75 \%, 33 \%, 36.4 \%, 38.5 \%$, and $50 \%$ lost more than $50 \%$ of EWL at $6,12,18,24$, and 30 months after surgery, respectively.

Eight most common comorbidities were assessed and they were present in $59.5 \%(N=50)$ of patients before surgery. Complete resolution of one comorbid condition was observed in 23 cases and improvement or significant improvement was observed in 21 cases. As the most common, arterial hypertension improved or resolved in $62 \%$ of cases, and diabetes mellitus improved or resolved in
$68.3 \%$ of affected patients. Changes in all present comorbid conditions have been presented in Table 2.

Results of the Moorehead-Ardelt Quality of Life Questionnaire are shown in Table 3. The self-esteem and physical activity aspects of QoL have improved most significantly with interest in sex remaining in more than $50 \%$ of patients at the same level.

All surgical and medical complications were stratified as major and minor as well as early and late according to the Oria and Moorehead classification and were summarized in Table 4.

There was one conversion $(1.2 \%)$ to the open technique due to the massive bleeding that could have not been stopped with the laparoscopic approach and there were no reoperations in the first 30 postoperative days. During the follow-up period, three patients underwent another bariatric operation due to unsatisfactory weight loss $(1 \times$ resleeve, $2 \times$ Roux-en-Y gastric bypass).

Based on all the above factors, the global BAROS outcomes were calculated. Excellent outcome was achieved
Fig. 1 Changes of percentage of excess weight loss in time following LSG

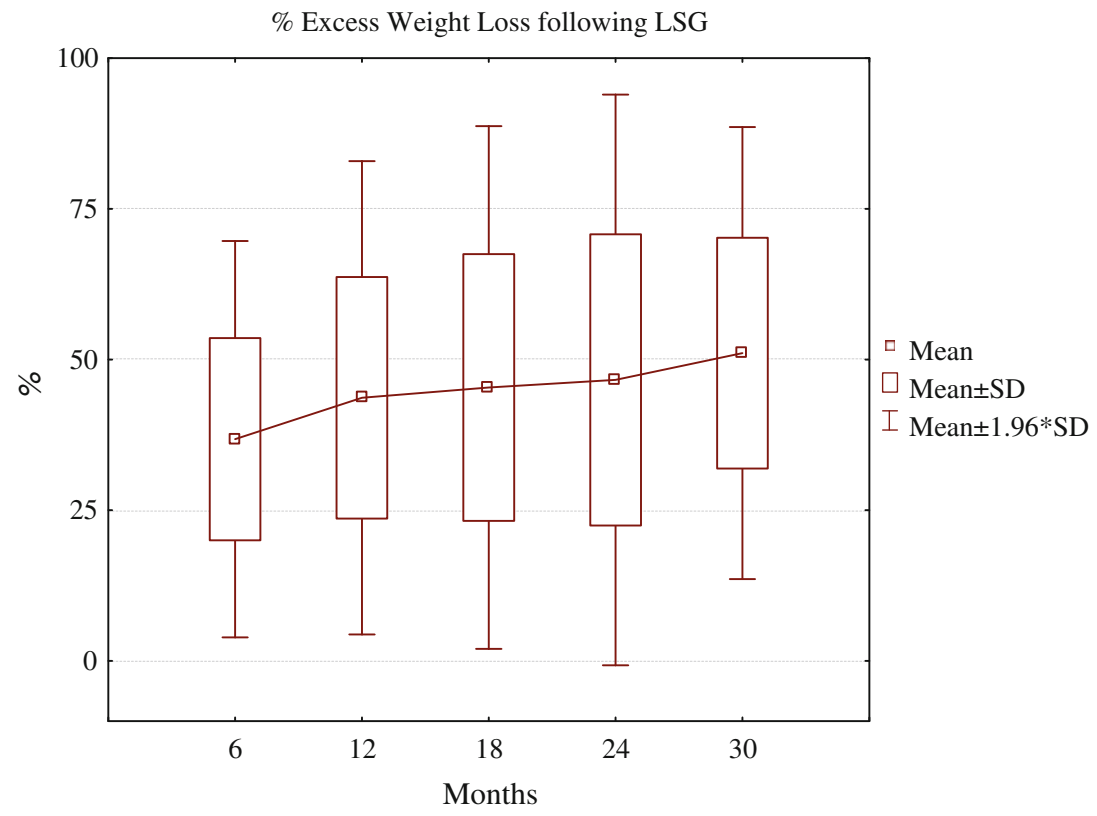


Table 2 Improvement and resolution of comorbidities $(N-$ responds to the number of affected patients - one patient could have more than one comorbidity)

\begin{tabular}{llllll}
\hline Co-morbidity & $\begin{array}{l}\text { Before surgery } \\
N\end{array}$ & $\begin{array}{l}\text { Improvement } \\
N(\%)\end{array}$ & $\begin{array}{l}\text { Resolution } \\
N(\%)\end{array}$ & $\begin{array}{l}\text { No change } \\
N(\%)\end{array}$ & $\begin{array}{l}\text { Worsening } \\
N(\%)\end{array}$ \\
\hline Arterial hypertension & 42 & $12(28.6)$ & $14(33.3)$ & $16(38)$ & 0 \\
Diabetes mellitus & 22 & $6(27.3)$ & $9(41)$ & $7(31.8)$ & 0 \\
Osteoarthritis & 9 & $1(11.1)$ & 0 & $8(89.9)$ & 0 \\
Asthma & 4 & $1(25)$ & 0 & $3(75)$ & 0 \\
Ischemic heart disease & 3 & 0 & 0 & $3(100)$ & 0 \\
Sleep apnoea & 1 & $1(100)$ & 0 & 0 & 0 \\
Thyroid disorders & 6 & 0 & 0 & $6(100)$ & 0 \\
Depression & 1 & 0 & 0 & $1(100)$ & 0 \\
\hline
\end{tabular}

in $11(13 \%)$ patients, very good in $25(30 \%)$, good in 29 $(34.5 \%)$, fair in eight $(9.5 \%)$, and failure in $11(13 \%)$ patients.

Further analysis has shown that females achieved significantly better outcomes than males (Table 5) with the mean $46.5 \%$ of EWL versus $35.3 \%$ of EWL at 12 months $(p=0.02)$. There was no statistically significant difference in outcomes in correlation to education $(p=0.17)$, smoking $(p=0.06)$, obesity of the first-degree relative ( $p=0.13)$, childhood obesity $(0.47)$, having had previous bariatric surgery $(p=0.59)$, declared increased physical activity $(p=0.96)$, and declared decreased calories intake and diet modification $(p=0.6)$.

The outline of the BAROS system excludes patients lost to follow-up or the ones who died during the operation or shortly postoperatively. Among 112 patients initially eligible for inclusion to the study, one female patient $(0.9 \%)$ died due to the pulmonary artery embolism on the fifth postoperative day therefore was excluded from final analysis.

\section{Discussion}

Among many well-established bariatric operations, LSG as a stand-alone operation is relatively new. Nevertheless, the operative technique is well-developed, established, and standardized [1]. In the available literature, there are few large series studies assessing its outcomes, and authors use multiple outcome measures and assessment instruments; therefore, the outcomes are difficult to compare [8-10].
BAROS give broader assessment of the outcome than the use of percentage of the excess weight loss on its own [7]. Among limitations of BAROS is exclusion of patients with perioperative deaths and the ones lost to follow-up.

In the presented series operative time of $61 \mathrm{~min}$ and hospital stay of 1.37 days were shorter than in the recent review by Shi et al. summarizing outcomes of 940 cases (100.4 min and 4.4 days, respectively) [8].

Of the patients, $74 \%$ ( $81 \%$ of females and $66.6 \%$ of males) have achieved scores from good to excellent using BAROS criteria. Of the operations, $13.1 \%$ in total were classified as failures due to the poor global BAROS scores. Those patients had poor weight loss or weight regain during the first 12 months following surgery with poor QoL scores and some postoperative complications. Three $(3.6 \%)$ of those patients consecutively underwent another bariatric operations.

In opposition to the single criterion outcome measures, BAROS shows global outcome apart from $\%$ EWL including also QoL, resolution of comorbidities, complications, and need for reoperations [7]. Therefore, the global impact of bariatric surgery is assumed greater than only percentage of excess weight loss. In the presented series, the mean percentage of the EWL of $43.6 \%$ at 12 months and $46.6 \%$ at 24 months of follow-up is lower than the expected mean $60 \%$ and $65 \%$ loss of EWL in 12 and 24 months in review by Shi et al. [8]. It is still much better than the results published by Regan et al. [11] and Milone et al. [12] with EWL reaching only 33-35\%. Nevertheless, the explanation of this fact could potentially lie in the poor adherence to the recommendations following surgery with only $32.9 \%$ of patients declaring increase of physical activity to at least
Table 3 Moorehead-Ardelt quality of life questionnaire results

\begin{tabular}{llcccc}
\hline & Much worse (\%) & Worse (\%) & Same (\%) & Better (\%) & Much better (\%) \\
\hline Self-esteem & $1(1.2)$ & $3(3.6)$ & $4(4.8)$ & $20(23.8)$ & $56(66.6)$ \\
Physical activity & 0 & $3(3.6)$ & $9(10.7)$ & $23(27.4)$ & $49(58.3)$ \\
Social involvement & 0 & $5(5.9)$ & $17(20.2)$ & $26(31)$ & $36(42.9)$ \\
Ability to work & 0 & $2(2.4)$ & $25(29.7)$ & $33(39.3)$ & $24(28.6)$ \\
Interest in sex & $3(3.6)$ & $3(3.6)$ & $44(52.4)$ & $16(19)$ & $18(21.4)$ \\
\hline
\end{tabular}


Table 4 Complications

Surgical complications

\begin{tabular}{|c|c|c|c|}
\hline \multicolumn{2}{|l|}{ Major $7.1 \%(N=6)$} & \multicolumn{2}{|l|}{ Minor $8.3 \%(N=7)$} \\
\hline Early $4.8 \%(N=4)$ & Late $2.4 \%(N=2)$ & Early $1.2 \%(N=1)$ & Late $7.1 \%(N=6)$ \\
\hline $\begin{array}{l}\text { Infarction of the upper splenic } \\
\text { pole } 2.4 \%(N=2)\end{array}$ & - Incisional hernia $2.4 \%(N=2)$ & $\begin{array}{l}\text { - Small wound infection } \\
1.2 \%(N=1)\end{array}$ & $\begin{array}{l}\text { Persistent nausea or vomiting } \\
4.8 \%(N=4)\end{array}$ \\
\hline - GI leak with peritonitis $1.2 \%(N=1)$ & & & - Stenosis of the stomach $1.2 \%(N=1)$ \\
\hline - Wound abscess $1.2 \%(N=1)$ & & & - Electrolyte imbalance $1.2 \%(N=1)$ \\
\hline
\end{tabular}

Medical complications

\begin{tabular}{|c|c|c|c|}
\hline \multicolumn{2}{|l|}{ Major $3.6 \%(N=3)$} & \multicolumn{2}{|l|}{ Minor $15.5 \%(N=13)$} \\
\hline Early $3.6 \%(N=3)$ & Late $0 \%$ & Early $4.8 \%(N=4)$ & Late $10.7 \%(N=9)$ \\
\hline - Depression $2.4 \%(N=2)$ & & - Atelectasis $1.2 \%(N=1)$ & - Hair loss $4.8 \%(N=4)$ \\
\hline \multirow[t]{3}{*}{ - Breathing disturbances $1.2 \%(N=1)$} & & - Vomiting $1.2 \%(N=1)$ & - Anemia 4.8\% $(N=4)$ \\
\hline & & $\begin{array}{l}\text { - Electrolyte imbalance } \\
1.2 \%(N=1)\end{array}$ & $\begin{array}{l}\text { - Metabolic deficiency } \\
\text { (protein, vitamins) } 1.2 \%(N=1)\end{array}$ \\
\hline & & $\begin{array}{l}\text { - Urinary tract infection } \\
1.2 \%(N=1)\end{array}$ & \\
\hline
\end{tabular}

30 min three times a week and as many as $55.7 \%$ of patients not having any physical activity postoperatively. According to the systematic review by Livhits et al., increase in physical activity to at least $30 \mathrm{~min}$ a day, three times a week might be associated with decrease of BMI by as much as $4 \%$ of initial BMI [13]. Interestingly, as many as $79.7 \%$ of patients declared change of their diet with significant calories and carbohydrates intake reduction following surgery, which did not correspond to $\% \mathrm{EWL}$ and the global outcomes $(p=0.6)$. In the presented series, the LSG had greater influence on the body mass reduction in females with statistically significant higher percentage of excess weight loss at 12 months after surgery $(46.5 \% \mathrm{EWL}$ versus $35.3 \% \operatorname{EWL}(p=0.02))$ and thanks to that fact they achieved better outcomes than males. Most probably, this difference is based mainly on signifi-

Table 5 Outcome based on gender

\begin{tabular}{lcc}
\hline & \multicolumn{2}{l}{ Gender } \\
\cline { 2 - 3 } Outcome & $\begin{array}{l}\text { Female } \\
N=63(100 \%)\end{array}$ & $\begin{array}{l}\text { Male } \\
N=21(100 \%)\end{array}$ \\
\hline Excellent & $11(17.5)$ & 0 \\
Very good & $21(33.3)$ & $4(19)$ \\
Good & $19(30.2)$ & $10(47.6)$ \\
Fair & $5(7.9)$ & $3(14.3)$ \\
Failure & $7(11.1)$ & $4(19.1)$ \\
\hline
\end{tabular}

cantly lower initial BMI. Nevertheless, the data collected during the study did not allow the analysis that would show its background. If the above conclusion is true, one might attempt a further conclusion that the LSG as a stand-alone procedure is more effective in 'slimmer' patients with lower initial BMI but to prove it further large case controlled studies should be performed. The number of super morbid obesity patients was reduced from $21.4 \%(N=18)$ to $3.6 \%(N=3)$, although the weight loss was more limited in this group than in the group of patients with lower preoperative BMI.

The well-known positive outcome of metabolic surgery is resolution or improvement of comorbid medical conditions [14]. Eight most common comorbidities were assessed and they were present in $59.5 \%(N=50)$ of patients preoperatively. Complete resolution of one comorbid condition was observed in 23 cases and improvement or significant improvement was observed in 21 cases. Improvement or resolution in $62 \%$ of cases of arterial hypertension and $68.3 \%$ of diabetes mellitus cases is just the same as achieved by Basso et al. in their large series of 300 cases of LSG [9].

The quality-of-life assessment incorporated in BAROS scale assumes changes of QoL in time following bariatric surgery and compares current state with baseline from before the surgery. Major positive changes in QoL were reported in self-esteem and physical activity domains with some improvement in social involvement and ability to work and the moderate improvement in the interest in sex.

Major early surgical complications (listed by the BAROS questionnaire) in the presented series reached $4.8 \%$, which is a 
good result when compared to $12.1 \%$ in the review by Shi et al. [8] and $9 \%$ in the single institution study by Basso et al. [9]. On the contrary, upper splenic pole infarction was the most frequent complication (two patients), followed by one staples line leak and one wound abscess.

Interestingly, $10.7 \%$ of patients developed late medical complications uncommon for restrictive bariatric procedures but typical for malabsorptive procedures with anemia, hair loss, and metabolic deficiencies including proteins and vitamins deficiencies [15]. The study design did not allow identification of the causative factors.

Out of the 112 patients initially qualified into the study and operated on, one female patient $(0.9 \%)$ died due to the pulmonary artery embolism on the fifth postoperative day despite antithrombotic prophylaxis. This patient was not included in the outcomes analysis, as those patients are excluded accordingly to the BAROS scale design. Mortality was slightly higher than the mortality in other LSG studies $0.5 \%$ [16] and $0.3 \%$ [8]; nevertheless, surgery-related mortality decreases with the center's bariatric experience.

\section{Conclusions}

The presented series of laparoscopic sleeve gastrectomies shows that it provides acceptable percentage of weight loss and good global BAROS outcomes. It significantly improves comorbidities. However, its metabolic impact still needs further studies to explain deficiencies typical for malabsorptive procedures.

Conflict of interest The authors declare that they have no conflict of interest.

Open Access This article is distributed under the terms of the Creative Commons Attribution Noncommercial License which permits any noncommercial use, distribution, and reproduction in any medium, provided the original author(s) and source are credited.

\section{References}

1. Iannelli A, Dainese $\mathrm{R}$, Piche $\mathrm{T}$, et al. Laparoscopic sleeve gastrectomy for morbid obesity. World J Gastroenterol. 2008;14 (6):821-7. 14.

2. Hess DS, Hess DW. Biliopancreatic diversion with a duodenal switch. Obes Surg. 1998;8:267-82.

3. Ren CJ, Patterson E, Gagner M. Early results of laparoscopic biliopancreatic diversion with duodenal switch: a case series of 40 consecutive patients. Obes Surg. 2000;10:514-23.

4. Ware JE, Sherbourne CD. The MOS 36-item short-form health survey (SF-36) I: conceptual framework and item selection. Med Care. 1992;30:473-83.

5. Karlsson J, Sjöström L, Sullivan M. Swedish obese subjects (SOS) - an intervention study of obesity. Two-year-follow-up of health-related quality of life (HRQL) and eating behavior after gastric surgery for severe obesity. Int J Obes Relat Metab Disord. 1998;22:113-26.

6. Kaplan RM, Bush JW. Health-related quality of life measurement for evaluation research and policy analysis. Health Psychol. 1982;1(1):61-80.

7. Oria HE, Moorehead MK. Bariatric Analysis and Reporting Outcome System (BAROS). Obes Surg. 1998;8:487-99.

8. Shi X, Karmali S, Sharma AM, et al. A review of laparoscopic sleeve gastrectomy for morbid obesity. Obes Surg. 2010;20:1171-7.

9. Basso N, Casella G, Rizzello M, et al. Laparoscopic sleeve gastrectomy as first stage or definitive intent in 300 consecutive cases. Surg Endosc. 2011;25(2):444-9.

10. Karmali S, Schauer P, Birch D, et al. Laparoscopic sleeve gastrectomy: an innovative new tool in the battle against the obesity epidemic in Canada. Can J Surg. 2010;53(2):126-32.

11. Regan JP, Inabnet WB, Gagner M. Early experience with two stage laparoscopic Roux-en-Y gastric bypass as an alternative in the super-super obese patient. Obes Surg. 2003;13(6):861-4.

12. Milone L, Strong V, Gagner M. Laparoscopic sleeve gastrectomy is superior to endoscopic intragastric balloon as a first-stage procedure for super-obese patients $(B M I \geq 50)$. Obes Surg. 2005; 15(5):612-7.

13. Livhits M, Mercado C, Yermilov I, et al. Exercise following bariatric surgery: systematic review. Obes Surg. 2010;20:657-65.

14. Sjöström L, Lindroos AK, Peltonen M, et al. Lifestyle, diabetes, and cardiovascular risk factors 10 years after bariatric surgery. $\mathrm{N}$ Engl J Med. 2004;351:2683-93.

15. Alvarez-Leite JI. Nutrient deficiencies secondary to bariatric surgery. Curr Opin Clin Nutr Metab Care. 2004;7(5):569-75.

16. Frezza EE, Reddy S, Gee LL, et al. Complications after sleeve gastrectomy for morbid obesity. Obes Surg. 2009;19:684-7. 